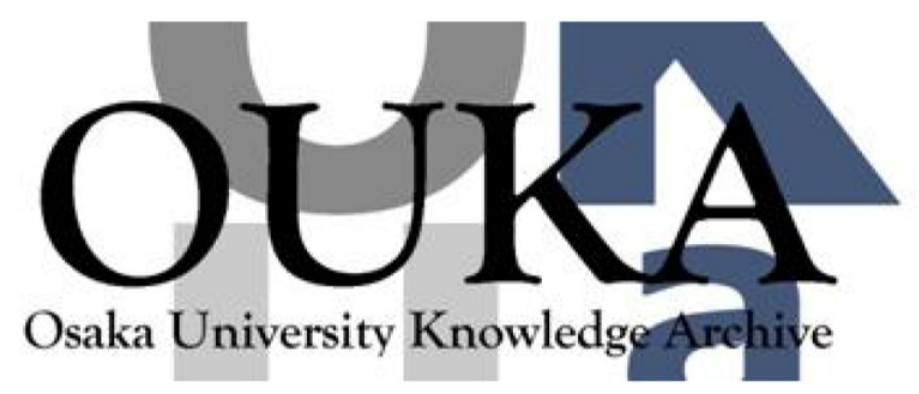

\begin{tabular}{|c|l|}
\hline Title & $\begin{array}{l}\text { Cherenkov radiation generated by a beam of } \\
\text { electrons revisited }\end{array}$ \\
\hline Author(s) & Zheng, Jian; Yu, C.X.; Zheng, Z.J. et al. \\
\hline Citation & Physics of Plasmas. 12(9) p. 093105 \\
\hline Issue Date & $2005-09$ \\
\hline oaire:version & VoR \\
\hline URL & https://hdl. handle. net/11094/2989 \\
\hline rights & \\
\hline Note & \\
\hline
\end{tabular}

Osaka University Knowledge Archive : OUKA

https://ir. Library. osaka-u. ac. jp/

Osaka University 


\title{
Cherenkov radiation generated by a beam of electrons revisited
}

\author{
Jian Zheng ${ }^{\text {a) }}$ and C. X. Yu \\ CAS Key Laboratory of Basic Plasma Physics and Department of Modern Physics, \\ University of Science and Technology of China, Hefei, Anhui 230026, People's Republic of China \\ Z. J. Zheng \\ Research Center of Laser Fusion, China Academy of Engineering Physics, Mianyang, Sichuan 621900, \\ People's Republic of China \\ K. A. Tanaka \\ Institute of Laser Engineering, Osaka University, Yamada-oka 2-6, Suita, Osaka 565-0871, Japan
}

(Received 25 May 2005; accepted 26 July 2005; published online 2 September 2005)

Cherenkov radiation generated by a beam of electrons is theoretically investigated. In the case that the boundary effect is negligible, coherent Cherenkov radiation does not depend on the longitudinal bunch form of the electron beam, which is remarkably different from other kinds of coherent radiation like coherent transition radiation and coherent synchrotron radiation. The reason for this result is ascribed to the criterion of the emission of Cherenkov radiation. The angular distribution of coherent Cherenkov radiation is mainly determined by the transverse bunch form of the beam. The spectral intensity of incoherent Cherenkov radiation is proportional to the velocity distribution function of the electrons in the beams. Based on these results, some methods are suggested to study hot electrons with the measurement of Cherenkov radiation. (c) 2005 American Institute of Physics. [DOI: $10.1063 / 1.2040178]$

\section{INTRODUCTION}

In recent years there has been a growing interest in coherent radiation from short bunches of electrons, either as a source of millimeter and submillimeter waves or as a highresolution monitor of the bunch form. Several kinds of coherent radiation have been investigated, such as coherent synchrotron radiation ${ }^{1-3}$ and coherent transition radiation. ${ }^{4-8}$ Very recently, the investigation of coherent radiation extends to the field of ultraintense laser matter interactions. By measuring coherent transition radiation emitted from the rear surface of a foil target, the heating mechanisms and the temperature of the hot electrons generated in ultraintense laser matter interactions are inferred. ${ }^{9,10}$ The propagation of hot electrons inside a target is a more attractive topic because it is closely related to the fast igniter scheme of inertial fusion energy. ${ }^{11}$ Direct measurement of the propagation of hot electron beams inside a target is a challenge because the trajectories of hot electrons are usually unobservable. A possible access to the study of the propagation of hot electrons inside a target could be realized by using a transparent target. Since high-energy electrons can emit light waves due to Cherenkov radiation, measurement of Cherenkov radiation generated by a beam of hot electrons could provide an approach to this subject, even though this method is restricted to the case that the target materials should be transparent.

Although Cherenkov radiation has been well known for a long time, coherent Cherenkov radiation has been experimentally investigated by several authors only in recent years. ${ }^{12-14}$ Due to finite path length of energetic charges in a medium, boundary effects like transition radiation may also contribute to the observation. For example, the observation

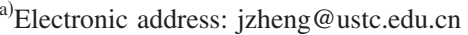

of coherent Cherenkov radiation reported in Ref. 12 is finally ascribed to transition radiation but not Cherenkov radiation because the path length of the high-energy electrons in the medium is smaller than the formation zone. ${ }^{14}$ The effect of finite path length on Cherenkov radiation was first investigated by Tamm, ${ }^{15}$ and later by several other authors. ${ }^{16-18} \mathrm{In}$ this article, we revisit Cherenkov radiation generated by a beam of electrons, with the inclusion of finite path length and coherent effects. We find that the spectral intensity of incoherent Cherenkov radiation is proportional to the velocity distribution of the electrons in the beam, and that the angular distribution of coherent Cherenkov radiation is largely determined by the form of the beam cross section. The most remarkable conclusion of our calculations is that neither the longitudinal bunch form nor the microbunching of the beam has impact upon coherent Cherenkov radiation when the path length is much longer than the radiation wavelength. Based on these results, we suggest some methods for studying hot electrons with the measurement of Cherenkov radiation.

\section{CALCULATIONS AND DISCUSSIONS}

\section{A. Basic equations}

We start our discussion from the Cherenkov radiation generated by single electron. The radiation can be described with the following equation: ${ }^{19}$

$$
\nabla^{2} \mathbf{A}-\frac{\hat{\epsilon}}{c^{2}} \frac{\partial^{2} \mathbf{A}}{\partial t^{2}}=-\frac{4 \pi}{c} \mathbf{j} .
$$

Here $\mathbf{j}$ is the electric current, $\hat{\boldsymbol{\epsilon}}$ is the operator of the permittivity $\epsilon$ : $\hat{\epsilon} \exp (-i \omega t)=\epsilon(\omega) \exp (-i \omega t)$, and $\mathbf{A}(\mathbf{r}, t)$ is the vector potential, from which the magnetic field $\mathbf{H}$ of the radia- 
tion can be obtained, $\mathbf{H}=\nabla \times \mathbf{A}$. We expand $\mathbf{A}$ as a Fourier time integral,

$$
\mathbf{A}(\mathbf{R}, t)=\int_{-\infty}^{\infty} \mathbf{A}_{\omega}(\mathbf{R}) e^{-i \omega t} \frac{d \omega}{2 \pi}
$$

The Fourier component of the vector potential is then given by

$$
\left.\mathbf{A}_{\omega}(\mathbf{R})=\frac{1}{c} \int \frac{\mathbf{j}_{\omega}(\mathbf{r})}{|\mathbf{R}-\mathbf{r}|} \exp [i \omega|\mathbf{R}-\mathbf{r}| \sqrt{\epsilon} / c)\right] d^{3} r
$$

where $\mathbf{j}_{\omega}(\mathbf{r})$ is the Fourier component of the electric current j. In wave zone far from the particle, i.e., $R \gg r$, we approximately have $|\mathbf{R}-\mathbf{r}| \approx R-\mathbf{n} \cdot \mathbf{r}$, where $\mathbf{n}=\mathbf{R} / R$. The Fourier component of the vector potential can be approximated as

$$
\mathbf{A}_{\omega}=\frac{\exp (i k R)}{c R} \int \mathbf{j}_{\omega} \exp (-i \mathbf{k} \cdot \mathbf{r}) d^{3} r
$$

where $\mathbf{k}=(\omega / c) \epsilon^{1 / 2} \mathbf{n}$. For an electron, the current can be written as

$$
\mathbf{j}(t)=-e \mathbf{v}(t) \delta\left[\mathbf{r}-\mathbf{r}_{0}(t)\right],
$$

where $\delta(x)$ is the $\delta$ function, $\mathbf{r}_{0}(t)$ is the trajectory of the electron, and $e$ is the electron charge. Substituting the Fourier component of the electric current

$$
\mathbf{j}_{\omega}(\mathbf{r})=-e \int_{-\infty}^{\infty} \mathbf{v}(t) \delta\left[\mathbf{r}-\mathbf{r}_{0}(t)\right] e^{i \omega t} d t
$$

into Eq. (4), and integrating over the volume $d^{3} r$, we obtain the relation between the vector potential and the electron movement,

$$
\mathbf{A}_{\omega}=-\frac{e}{c R} e^{i k R} \int_{-\infty}^{\infty} \mathbf{v}(t) \exp \left[i \omega t-i \mathbf{k} \cdot \mathbf{r}_{0}(t)\right] d t .
$$

The magnetic field of the radiation is then given by

$$
\mathbf{H}_{\omega} \approx-\frac{i e k}{c R} e^{i k R} \int_{-\infty}^{\infty} \mathbf{n} \times \mathbf{v}(t) e^{i\left[\omega t-\mathbf{k} \cdot \mathbf{r}_{0}(t)\right]} d t .
$$

Here we just keep the term proportional to $1 / R$.

The radiation energy across the section $\mathbf{n} R^{2} d \Omega$ in wave zone is given by

$$
\begin{aligned}
d \mathcal{E} & =\frac{c}{4 \pi}\left(\int_{0}^{\infty}(\mathbf{E} \times \mathbf{H}) \cdot \mathbf{n} d t\right) R^{2} d \Omega \\
& =\frac{c}{4 \pi^{2}}\left(\int_{0}^{\infty} \frac{1}{\epsilon(\omega)^{1 / 2}}\left|\mathbf{H}_{\omega}\right|^{2} d \omega\right) R^{2} d \Omega .
\end{aligned}
$$

The spectral intensity of the radiation is described by

$$
\begin{aligned}
\frac{d^{2} \mathcal{E}}{d \omega d \Omega}= & \frac{c}{4 \pi^{2}} \frac{R^{2}}{\sqrt{\epsilon(\omega)}}\left|\mathbf{H}_{\omega}\right|^{2}=\frac{e^{2} \omega^{2} \sqrt{\epsilon}}{4 \pi^{2} c^{3}} \mid \int_{-\infty}^{\infty} \mathbf{n} \\
& \times\left.\mathbf{v}(t) e^{i\left[\omega t-\mathbf{k} \cdot \mathbf{r}_{0}(t)\right]} d t\right|^{2}
\end{aligned}
$$

The size of a medium is actually finite. Therefore, the trajectory of a charge inside the medium is finite, too. We

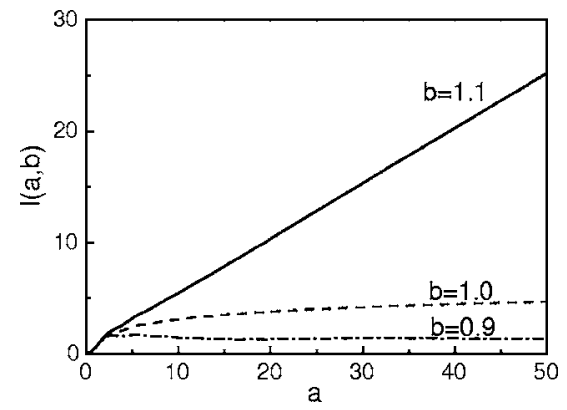

FIG. 1. The integral $I(a, b)$ vs the variable $a$ when $b=1.1,1.0$, and 0.9, where $a=\omega L / 2 \beta c$ and $b=\beta n$.

assume that the charge moves in the medium during the time from $t=\tau$ to $t=\tau+L / v$, where $L$ is the length of the trajectory. In this case, the spectral radiation intensity is given by

$$
\frac{d^{2} \mathcal{E}}{d \omega d \Omega}=\frac{e^{2} \beta^{2} n \sin ^{2} \theta}{\pi^{2} c} \frac{\sin ^{2}[(\omega L / 2 \beta c)(1-\beta n \cos \theta)]}{(1-\beta n \cos \theta)^{2}},
$$

where $\beta=v / c, n=\epsilon^{1 / 2}$ is the refractive index of the medium, and $\theta$ is the angle between the wave vector $\mathbf{k}$ and the particle velocity v. As seen in Eq. (7), there exists some radiation even though the critical condition of the Cherenkov radiation, $\beta n>1$ is not satisfied. This fact is due to our assumption with which we obtain Eq. (7). Since the trajectory of the charge is finite, the charge is assumed to be suddenly accelerated at the end points of the trajectory. This assumption could be properly expressed as

$$
\frac{d \mathbf{v}(t)}{d t}=\mathbf{v}[\delta(t-\tau)-\delta(t-\tau-L / v)]
$$

Of course, bremsstrahlung radiation should be emitted due to the acceleration. As pointed out by Aitken et al., ${ }^{16}$ a finite trajectory is also equivalent to considering a finite length of the medium terminated with a pair of metal surfaces but without considering the effect of image charges. From this point of view, the radiation can also been regarded as transition radiation occurring when the electron leaves and enters the metals. However, since the effect of image charges is not included in Eq. (8), the radiation cannot be simply described with the usual theory of transition radiation. This boundary effect can be seen more clearly by integrating Eq. (7) over the solid angle $d \Omega$. For the sake of simplicity, we introduce the notation $I(a, b)$, which is defined as

$$
I(a, b)=\int_{0}^{\pi / 2} \frac{\sin ^{2}[a(1-b \cos \theta)]}{(1-b \cos \theta)^{2}} \sin ^{3} \theta d \theta .
$$

Equation (7) can then be rewritten as

$$
\frac{d \mathcal{E}}{d \omega}=\frac{2 e^{2} \beta^{2} n}{\pi c} I(\omega L / 2 \beta c, \beta n) .
$$

In Fig. 1, we plot the integral $I(a, b)$ versus the variable $a$ for $b=1.1,1.0$, and 0.9 . As clearly seen in this figure, the integral $I(a, b)$ sensitively depends on the parameter $b$. When $a \gg 1$, the asymptotic expansion of the integral $I(a, b)$ depends on the parameter $b$, i.e., 
$I(a, b)=\left\{\begin{array}{l}a \pi\left(b^{2}-1\right) / b^{3}-\left[\ln (b-1)+b+b^{2} / 2\right] / b^{3}+O(1 / a), \text { when } b>1 \\ \ln (2 a)+\gamma-1 / 2, \text { when } b=1, \\ -\left[\ln (1-b)+b+b^{2} / 2\right] / b^{3}+O(1 / a), \text { when } b<1\end{array}\right.$

where $\gamma=0.577 \ldots$ is the Euler constant. Using this asymptotic expansion, we obtain the spectral energy in the case of $\omega L / 2 \beta c \gg 1$ and $\beta n>1$,

$$
\begin{aligned}
\frac{d \mathcal{E}}{d \omega}= & -\frac{2 e^{2} \beta}{\pi c(\beta n)^{2}}\left(\ln (\beta n-1)+\beta n+\frac{1}{2} \beta^{2} n^{2}\right)+\frac{e^{2} \omega L}{c^{2}}(1 \\
& \left.-\frac{1}{\beta^{2} n^{2}}\right)+O\left(\frac{\beta c}{\omega L}\right) .
\end{aligned}
$$

The first term on the right-hand side of Eq. (11) is independent of the trajectory. This term is due to the boundary condition of Eq. (8) that we choose. As mentioned above, however, the effect of the image charges is not included in our calculations. So the first term on the right-hand side of Eq. (11) does not simply reduce to the result of transition radiation. The second term on the right-hand side of the equation is the well-known Cherenkov radiation. It is proportional to the length of the charge trajectory. In the condition of $\omega L / 2 \beta c \gg 1$ and $\beta n>1$, the first term on the right-hand side of Eq. (11) can be neglected in comparison with the second term. As seen in Fig. 1 , for $b=1.1$, the integral $I(a, b)$ is nearly proportional to $a$ when $a \gg 1$. This result indicates that in an actual experiment of Cherenkov radiation the contribution of boundary effect could be negligible with a proper experimental setup.

When a beam of electrons passes through a transparent medium, Cherenkov radiation can be separated into two parts: one part is incoherent radiation, the other is coherent radiation. ${ }^{20}$ The former is simply the summation of the radiation due to individual electrons. The latter is determined by the interferences between the radiation fields generated by different electrons. The incoherent spectral radiation intensity is

$$
\frac{d^{2} \mathcal{E}_{I}}{d \omega d \Omega}=\frac{e^{2} \omega^{2} n}{4 \pi^{2} c^{3}} \sum_{i=1}^{N}\left|\int_{-\infty}^{\infty} \mathbf{n} \times \mathbf{v}_{i}(t) e^{i\left[\omega t-\mathbf{k} \cdot \mathbf{r}_{i}(t)\right]} d t\right|^{2},
$$

and the coherent spectral radiation intensity is

$$
\begin{aligned}
\frac{d^{2} \mathcal{E}_{\mathrm{C}}}{d \omega d \Omega}= & \frac{e^{2} \omega^{2} n}{4 \pi^{2} c^{3}} \sum_{\substack{i, j=1 \\
(i \neq j)}}^{N}\left(\int_{-\infty}^{\infty} \mathbf{n} \times \mathbf{v}_{i}(t) e^{i\left[\omega t-\mathbf{k} \cdot \mathbf{r}_{i}(t)\right]} d t\right) \\
& \times\left(\int_{-\infty}^{\infty} \mathbf{n} \times \mathbf{v}_{j}(t) e^{i\left[\omega t-\mathbf{k} \cdot \mathbf{r}_{j}(t)\right]} d t\right)^{*} .
\end{aligned}
$$

\section{B. Incoherent Cherenkov radiation}

We make some assumptions in order to perform further analytical calculations. We assume that the $i$ th electron starts to move uniformly with the velocity $v_{i}$ along the $z$ direction at the time $\tau_{i}$, and suddenly stops at the time $\tau_{i}+L / v_{i}$, and that the $i$ th electron originally locates at the point of $\left(\boldsymbol{\rho}_{i}, 0\right)$, i.e., all of the electrons start moving from the plane of $z=0$. With these assumptions, every electron is fully characterized with the set of the parameters $\left(\tau_{i}, \boldsymbol{\rho}_{i}, v_{i}\right)$. We introduce a distribution function to describe the electrons in the beam,

$$
f(\tau, \boldsymbol{\rho}, \mathrm{v})=\left\langle\frac{1}{N} \sum_{i}^{N} \delta\left(\tau-\tau_{i}\right) \delta\left(\boldsymbol{\rho}-\boldsymbol{\rho}_{i}\right) \delta\left(v-v_{i}\right)\right\rangle .
$$

Here the average $\langle\cdots\rangle$ in Eq. (14) can be consider as an ensemble average. In this case, we image that an infinite series of electron beams is created under the identical condition.

With aid of the distribution function (14), we find that the incoherent spectral intensity just depends on the velocity distribution of the electrons,

$$
\begin{aligned}
\frac{d^{2} \mathcal{E}_{I}}{d \omega d \Omega}= & N \frac{e^{2} n \sin ^{2} \theta}{\pi^{2} c} \int \frac{\sin ^{2}[(\omega L / 2 \beta c)(1-\beta n \cos \theta)]}{(1-\beta n \cos \theta)^{2}} \\
& \times \beta^{2} F_{\mathbf{v}}(v) d v,
\end{aligned}
$$

where $F_{\mathbf{v}}(v)$ is the velocity distribution function of the electrons given by

$$
F_{\mathbf{v}}(v)=\int f(\tau, \boldsymbol{\rho}, v) d \tau d^{2} \rho .
$$

In the case of $\omega L / 2 \beta c \gg 1$ and $\beta n>1$, substituting the formula

$$
\frac{\sin ^{2}[(\omega L / 2 \beta c)(1-\beta n \cos \theta)]}{(1-\beta n \cos \theta)^{2}}=\pi \frac{\omega L}{2 \beta c} \delta(1-\beta n \cos \theta),
$$

into Eq. (15), and integrating over velocity, we obtain

$$
\frac{d^{2} \mathcal{E}_{I}}{d \omega d \Omega}=N \omega L \frac{e^{2} \tan ^{2} \theta}{2 \pi n c} F_{\mathbf{v}}\left(\frac{c}{n \cos \theta}\right) .
$$

Equation (18) clearly shows that the spectral intensity of incoherent Cherenkov radiation is proportional to the velocity distribution function of electrons in a beam. This result indicates that the velocity distribution could be partially inferred from the spectrum of incoherent Cherenkov radiation. As we know, bulk accelerated electrons are usually mild relativistic in ultraintense laser matter interactions. These electrons could be investigated by using those materials with high refractive indices. For example, the refractive index of diamond varies from 2.38 to 2.79 when photon energy changes from 0.5 to $5.8 \mathrm{eV} .^{21}$ If a detector is set at the angle of $45^{\circ}$ to the beam direction and a photon energy spectrum is measured in the range from 0.5 to $5.8 \mathrm{eV}$, the velocity dis- 
tribution of a beam of electrons can be inferred in the range from $0.5087 c$ to $0.5942 c$. It should be pointed out that measurement of electron velocity distribution function with incoherent Cherenkov radiation may be superior to that with an electron spectrometer. This reason is that electrons are strongly affected by electric field due to charge separation at the rear surface of a target as they escape from the target and reach to an electron spectrometer. Such a factor would have no influence on the spectrum of Cherenkov radiation since Cherenkov radiation is emitted only when charges move inside a target.

\section{Coherent Cherenkov radiation}

We are also interested in coherent radiation. Since the electrons are assumed to move independently, the spectral intensity of coherent Cherenkov radiation is given by

$$
\begin{aligned}
\frac{d^{2} \mathcal{E}_{C}}{d \omega d \Omega}= & N(N-1) \frac{e^{2} n \sin ^{2} \theta}{\pi^{2} c} \mid \\
& \times \int \frac{\sin [(1-\beta n \cos \theta) \omega L / 2 \beta c]}{(1-\beta n \cos \theta)} \beta F[\omega(1 \\
& -\beta n \cos \theta), \mathbf{q}, v]\left.e^{i \omega L / 2 \beta c} d v\right|^{2},
\end{aligned}
$$

where the function $F(\omega, \mathbf{q}, v)$ is the Fourier component of the distribution function,

$$
F(\omega, \mathbf{q}, v)=\int e^{i \omega \tau-i \mathbf{q} \cdot \boldsymbol{\rho}} f(\tau, \boldsymbol{\rho}, v) d \tau d^{2} \rho,
$$

and $\mathbf{q}$ is the projection of the wave vector $\mathbf{k}$ in the $x-y$ plane, and $q=k \sin \theta$.

The effect of the bunch form of a beam on coherent spectrum of Cherenkov radiation is characterized with the function $F(\omega, \mathbf{q}, v)$, as clearly seen in Eq. (19). As an example, we first investigate the effect of bunch form in the case that a beam of monochromatic electrons has a Gaussian cross section. In this case, the distribution function of the electrons in the beam can be expressed as

$$
f(\tau, \boldsymbol{\rho}, v)=\frac{1}{(2 \pi)^{3 / 2} a^{2} \tau_{0}} e^{-\rho^{2} / 2 a^{2}} e^{-\tau^{2} / 2 \tau_{0}^{2}} \delta\left(v-v_{0}\right),
$$

where $a$ is the radius of the cross section of the beam and $\tau_{0}$ is the pulse duration of the beam. Substituting the distribution function (21) into Eq. (20), we have

$$
F(\omega, \mathbf{q}, v)=e^{-q^{2} a^{2} / 2} e^{-\omega^{2} \tau_{0}^{2} / 2} \delta\left(v-v_{0}\right) .
$$

The spectral intensity of coherent radiation is then given by

$$
\begin{aligned}
\frac{d^{2} \mathcal{E}_{C}}{d \omega d \Omega}= & N(N-1) e^{-k^{2} a^{2} \sin ^{2} \theta} e^{-\omega^{2}\left(1-\beta_{0} n \cos \theta\right)^{2} \tau_{0}^{2} \beta_{0}^{2}} \\
& \times \frac{e^{2} n \sin ^{2} \theta}{\pi^{2} c} \frac{\sin ^{2}\left[\left(1-\beta_{0} n \cos \theta\right) \omega L / 2 \beta_{0} c\right]}{\left(1-\beta_{0} n \cos \theta\right)^{2}} .
\end{aligned}
$$

Here the factor $\exp \left(-k^{2} a^{2} \sin ^{2} \theta\right)$ is due to the transverse bunch form of the beam, and the factor $\exp \left[-\omega^{2}(1\right.$ $\left.\left.-\beta_{0} n \cos \theta\right)^{2} \tau_{0}^{2}\right]$ is due to the longitudinal one. In the case that $\beta_{0} n>1$ and $\omega L / 2 \beta_{0} c \gg 1$, we can use the approximation of Eq. (17). Then we have

$$
\begin{aligned}
\frac{d^{2} \mathcal{E}_{C}}{d \omega d \Omega}= & N(N-1) \omega L \exp \left(-k^{2} a^{2} \sin ^{2} \theta\right) \\
& \times \frac{e^{2} n \sin ^{2} \theta}{2 \pi c^{2}} \beta_{0} \delta\left(1-\beta_{0} n \cos \theta\right) .
\end{aligned}
$$

Now the term due to the longitudinal bunch form, $\exp \left[-\omega^{2}\left(1-\beta_{0} n \cos \theta\right)^{2} \tau_{0}^{2}\right]$ disappears because of the $\delta$ function in Eq. (24). Only the term due to the transverse bunch form remains. This property of coherent Cherenkov radiation is remarkably different from other kinds of coherent radiation, such as coherent transition radiation and coherent synchrotron radiation in which both the longitudinal and transverse bunch forms are brought into effect. This result indicates that we could not investigate the longitudinal bunch form of a beam by measuring coherent Cherenkov radiation. It should be pointed out that Eq. (24) is obtained in the case that the approximation of Eq. (17) is valid, i.e., the conditions of $\beta_{0} n>1$ and $\omega L / 2 \beta_{0} c \gg 1$ are satisfied. If these conditions are not satisfied, the effect of the longitudinal bunch form does not disappear at all. However, if neither of these conditions is satisfied, the radiation is essentially contaminated by transition radiation.

It has been demonstrated that microbunching in an electron beam can profoundly affect the spectrum of coherent radiation like coherent transition radiation. ${ }^{9,10,20}$ Hot electron beams produced in ultraintense laser matter interactions can acquire microbunching due to the laser heating. The spectrum of coherent transition radiation thus presents intense harmonics corresponding to the microbunching in the beam. One may wonder if the microbunching can also affect coherent Cherenkov radiation. Here we address this question by modelling the microbunching in an electron beam with the following distribution function:

$$
f(\boldsymbol{\rho}, \tau, v)=\frac{1}{2 \pi a^{2}} e^{-\rho^{2} / 2 a^{2}} \delta\left(v-v_{0}\right) \frac{1}{\Lambda} \sum_{p=0}^{\Lambda-1} \delta\left(\tau-p \tau_{0}\right),
$$

where $\Lambda$ is the total number of microbunching in the beam, and $\tau_{0}$ is the time interval between two adjacent electron micropulses. For the electron beams generated in the ultraintense laser matter interactions, the value of $\tau_{0}$ is determined by the heating processes. ${ }^{9,10}$ With the distribution function of Eq. (25), the coherent spectral intensity is given by

$$
\begin{aligned}
\frac{d^{2} \mathcal{E}_{C}}{d \omega d \Omega}= & N(N-1) \frac{e^{2} \beta_{0}^{2} n}{\pi^{2} c} \frac{\sin ^{2}\left[\left(1-\beta_{0} n \cos \theta\right) \omega L / 2 v_{0}\right]}{\left(1-\beta_{0} n \cos \theta\right)^{2}} \\
& \times \frac{\sin ^{2}\left[\Lambda \omega \tau_{0}\left(1-\beta_{0} n \cos \theta\right) / 2\right]}{\Lambda^{2} \sin ^{2}\left[\omega \tau_{0}\left(1-\beta_{0} n \cos \theta\right) / 2\right]} \\
& \times \sin ^{2} \theta e^{-k^{2} a^{2} \sin ^{2} \theta .}
\end{aligned}
$$

Here the factor 


$$
\frac{\sin ^{2}\left[\Lambda \omega \tau_{0}\left(1-\beta_{0} n \cos \theta\right) / 2\right]}{\Lambda^{2} \sin ^{2}\left[\omega \tau_{0}\left(1-\beta_{0} n \cos \theta\right) / 2\right]}
$$

comes from the longitudinal microbunching of the beam. In the case of $\Lambda \gg 1$, this term has very sharp spikes at

$$
\omega \tau_{0}\left(1-\beta_{0} n \cos \theta\right) / 2=m \pi, \quad m=0, \pm 1, \pm 2, \cdots .
$$

If the criterion of Cherenkov radiation, $n \beta_{0}>1$, is not satisfied, the radiation spectrum shows sharp spikes at the harmonics corresponding to the microbunching in the beam, i.e.,

$$
\omega=\frac{m \omega_{0}}{1-\beta_{0} n \cos \theta}, \quad m=0, \pm 1, \pm 2, \cdots,
$$

where $\omega_{0}=2 \pi / \tau_{0}$. If it is true, we can obtain the characteristic frequency $\omega_{0}$ by measuring the spectrum of the radiation, and then infer the dominant heating process of the hot electrons. However, when the conditions $n \beta_{0}>1$ and $\omega L / 2 \beta_{0} c \gg 1$ are satisfied, only the frequency satisfying the condition $1-\beta_{0} n(\omega) \cos \theta=0$ is important due to the approximation of Eq. (17). In this case, the longitudinal factor of Eq. (27) becomes 1 because of

$$
\lim _{1-\beta_{0} n \cos \theta \rightarrow 0} \frac{\sin ^{2}\left[\Lambda \omega \tau_{0}\left(1-\beta_{0} n \cos \theta\right) / 2\right]}{\Lambda^{2} \sin ^{2}\left[\omega \tau_{0}\left(1-\beta_{0} n \cos \theta\right) / 2\right]}=1 .
$$

Then Eq. (26) is the same with Eq. (23) even though different bunching forms are assumed in the calculations.

The above discussions clearly show that the energy spectrum of coherent Cherenkov radiation does not depend on the longitudinal bunch form of an electron beam. Only the transverse bunch form of the beam can affect the radiation. Therefore, we cannot expect to investigate the bunch form of an electron beam by measurement of coherent Cherenkov radiation. The physical reason for this conclusion is that the emission of Cherenkov radiation is essentially governed by the criterion

$$
1-\beta n \cos \theta=0 .
$$

It is this criterion that totally removes the effect of the longitudinal bunch form. The effect of the longitudinal bunch form becomes notable only when Cherenkov radiation is not important. In this case, however, the radiation could be treated within the domain of transition radiation.

The effect of transverse bunch form on coherent Cherenkov radiation always exists. As shown in Eq. (24), The angular distribution of coherent radiation is largely determined by the transverse bunch form, i.e., the beam cross section. This result means that it is possible to measure the cross section of a hot electron beam inside a transparent target by detecting the angular distribution of coherent Cherenkov radiation.

\section{SUMMARY}

We have theoretically studied Cherenkov radiation generated by a beam of electrons. The most remarkable result of our research is that the spectral intensity of coherent Cherenkov radiation does not depend on the longitudinal bunch form of the beam. This result is very different from other kinds of coherent radiation like coherent transition ra- diation and coherent synchrotron radiation, in which the longitudinal bunch form has an important influence on coherent radiation. The transverse bunch form of a beam governs the angular distribution of coherent Cherenkov radiation. Thus the form of a beam cross section may be measured by detecting the angular distribution of coherent Cherenkov radiation. The spectral intensity of incoherent Cherenkov radiation is proportional to the velocity distribution of the electrons in a beams. Therefore, middle relativistic electrons could be studied with the measurement of the spectrum of incoherent Cherenkov radiation and with suitable target materials.

\section{ACKNOWLEDGMENTS}

This work is supported by the National Natural Science Foundation of China (Grant Nos. 10375064, 10275056, 10176028), the National High Technology Programs on Inertial Confinement Fusion, and the State Key Lab for High-Temperature High-Density Plasmas (Grant No. 51480010105ZK0101). J. Zheng is partially supported by the China-Japan Core University Programs of JSPS.

${ }^{1}$ J. S. Nodvick and D. S. Saxon, Phys. Rev. 96, 180 (1954).

${ }^{2}$ F. C. Michel, Phys. Rev. Lett. 48, 580 (1982).

${ }^{3}$ T. Nakazato, M. Oyamada, N. Niimura, S. Urasawa, O. Konno, A. Kagaya, R. Kato, T. Kamiyama, Y. Torizuka, T. Nanba, Y. Kondo, Y. Shibata, K. Ishi, T. Ohsaka, and M. Ikezawa, Phys. Rev. Lett. 63, 1245 (1989).

${ }^{4}$ U. Happek, A. J. Sievers, and E. B. Blum, Phys. Rev. Lett. 67, 2962 (1991).

${ }^{5}$ Y. Liu, X. J. Wang, D. B. Cline, M. Babzien, J. M. Fang, J. Gallardo, K. Kusche, I. Pogorelsky, J. Skaritka, and A. van Steenbergen, Phys. Rev. Lett. 80, 4418 (1998).

${ }^{6}$ A. Tremaine, J. B. Rosenzweig, S. Anderson, P. Frigola, M. Hogan, A. Murokh, C. Pellegrini, D. C. Nguyen, and R. L. Sheffield, Phys. Rev. Lett. 81, 5816 (1998).

${ }^{7}$ A. H. Lumpkin, R. Dejus, W. J. Berg, M. Borland, Y. C. Chae, E. Moog, N. S. Sereno, and B. X. Yang, Phys. Rev. Lett. 86, 79 (2001).

${ }^{8}$ A. H. Lumpkin, R. Dejus, J. W. Lewellen, W. Berg, S. Biedron, M. Borland, Y. C. Chae, M. Erdmann, Z. Huang, K.-J. Kim, Y. Li, S. V. Milton, E. Moog, D. W. Rule, V. Sajaev, and B. X. Yang, Phys. Rev. Lett. 88, 234801 (2002).

${ }^{9}$ S. D. Baton, J. J. Santos, F. Amiranoff, H. Popescu, L. Gremillet, M. Koenig, E. Martinolli, O. Guilbaud, C. Rousseaux, M. Rabec Le Gloahec, T. Hall, D. Batani, E. Perelli, and F. Scianitti, and T. E. Cowan, Phys. Rev. Lett. 91, 105001 (2003)

${ }^{10}$ J. Zheng, K. A. Tanaka, T. Sato, T. Yabuuchi, T. Kurahashi, Y. Kitagawa, R. Kodama, T. Norimatsu, and T. Yamanaka, Phys. Rev. Lett. 92, 165001 (2004).

${ }^{11}$ M. Tabak, J. Hammer, M. E. Glinsky, W. L. Kruer, S. C. Wilks, J. Woodworth, E. M. Campbell, M. D. Perry, and R. J. Mason, Phys. Plasmas 1, 1626 (1994).

${ }^{12}$ J. Ohkuma, S. Okuda, and K. Tsumori, Phys. Rev. Lett. 66, 1967 (1991).

${ }^{13}$ T. Takahashi, T. Kanai, Y. Shibata, K. Ishi, M. Ikezawa, T. Nakazato, M. Oyamada, S. Urasawa, T. Yamakawa, K. Takami, T. Matsuyama, K. Kobayashi, and Y. Fujita, Phys. Rev. E 50, 4041 (1994).

${ }^{14}$ T. Takahashi, Y. Shibata, K. Ishi, M. Ikezawa, M. Oyamada, and Y. Kondo, Phys. Rev. E 62, 8606 (2000).

${ }^{15}$ I. Tamm, J. Phys. (Moscow) 1, 439 (1939).

${ }^{16}$ D. K. Aitken, R. E. Jennings, A. S. Parsons, and R. N. F. Walker, Proc. Phys. Soc. London 82, 710 (1963).

${ }^{17}$ V. P. Zrelov, M. Klimannova, V. P. Pupiltsev, and J. Ružička, Nuclear Instruments and Methods 215, 141 (1983). 
${ }^{18}$ J. R. Neighbours, F. R. Buskirk, and X. Maruyama, J. Appl. Phys. 61, 2741 (1985).

${ }^{19}$ L. D. Landau and E. M. Lifshitz, Electrodynamics of Continuous Media, 2nd ed. (Butterworth-Heinemann, Oxford, 1984), Sect. 114.

${ }^{20}$ J. Zheng, K. A. Tanaka, T. Miyakoshi, Y. Kitagawa, R. Kodama, T.
Kurahashi, and T. Yamanaka, Phys. Plasmas 10, 2994 (2003); J. Zheng, K. A. Tanaka, T. Miyakoshi, Y. Kitagawa, R. Kodama, T. Kurahashi, and T. Yamanaka, Phys. Plasmas 9, 3610 (2002).

${ }^{21}$ The data are obtained from the website http://www.ioffe.ru/SVA/NSM/nk/ Miscellaneous. 